\title{
MAXIMA OF RANDOM ALGEBRAIC CURVES ${ }^{1}$
}

BY

\author{
M. DAS ${ }^{2}$ AND S. S. BHATT
}

\begin{abstract}
Let $X_{1}, X_{2}, \ldots, X_{n}$ be a sequence of independent and identically distributed random variables with common characteristic function $\exp \left(-|Z|^{\alpha}\right)$ where $0<\alpha<2$, and $P(x)=\sum_{1}^{n} X_{k} x^{k}$. Then we show that the numbers $M_{n}$ of maxima of the curves $y=P(x)$ have expectation $E M_{n} \sim c$ $\log n$, as $n \rightarrow \infty$, where $c=c(\alpha)=c_{1}(\alpha)+c_{2}(\alpha)$ and
\end{abstract}

$$
\begin{gathered}
c_{1}(\alpha)=\frac{1}{\pi^{2} \alpha^{2}} \int_{-\infty}^{\infty} d v \log \int_{0}^{\infty}\left[\frac{|v-y|^{\alpha}}{|v-1|^{\alpha}}\right] \exp (-y) d y, \\
c_{2}(\alpha)=\frac{1}{\pi^{2} \alpha^{2}} \int_{-\infty}^{\infty} \log \int_{0}^{\infty}\left\{\left(\frac{|v-z|^{\alpha}}{|v-\alpha-1|^{\alpha}}\right) \frac{z^{\alpha}}{\Gamma(1+\alpha)} \exp (-z) d z\right\} d v .
\end{gathered}
$$

1. Introduction. Let $X_{0}, X_{1}, \ldots, X_{n}$ be a sequence of independent and identically distributed random variables with a common characteristic function $\exp \left(-|z|^{\alpha}\right)$ where $0<\alpha<2$, and $P(x)=\Sigma_{0}^{n} X_{k} x^{k}$. The equation $y=P(x)$ defines an algebraic curve in the $x y$-plane corresponding to the random coefficients $\left(X_{0}, X_{1}, \ldots, X_{n}\right)$. We know from the works of Logan and Shepp [7], [8] that the members of this family, on an average, will cross the $x$-axis asymptotically $2 k_{0} \log n$ times when $n$ is large, where

$$
k_{0}=\frac{2}{\pi^{2} \alpha^{2}} \int_{-\infty}^{\infty} d x \log \left\{\int_{0}^{\infty}\left[\frac{|x-y|^{\alpha}}{|x-1|^{\alpha}}\right] \exp (-y) d y\right\}
$$

Clearly the algebraic curve $y=P(x)$ will have a number of maxima and minima determined by its turning points. Of course, a curve will have at least half as many maxima (minima) as it has crossings of the $x$-axis. We show that the number $M_{n}$ of maxima of the curves $y=P(x)$ have expectation

$$
E M_{n} \sim c \log n
$$

Received by the editors November 12, 1976.

AMS (MOS) subject classifications (1970). Primary 60G50, 60F99, 60D05.

Key words and phrases. Random variables, distribution, normally distributed random variables, sequence, mathematical expectation, variance, characteristic function, random algebraic curves.

${ }^{1}$ This constitutes a portion of the doctoral dissertation of the second author at the Utkal University.

${ }^{2}$ The first author was associated with the Mathematics Department at Ravenshaw College, Cuttack-753003, Orissa, India. 
as $n \rightarrow \infty$ where $c=c_{1}(\alpha)+c_{2}(\alpha)$ with

$$
c_{1}(\alpha)=\frac{1}{\pi^{2} \alpha^{2}} \int_{-\infty}^{\infty} d V \cdot \log \left[\int_{0}^{\infty}\left\{\frac{|V-y|^{\alpha}}{|V-1|^{\alpha}}\right\} \exp (-y) d y\right]
$$

and

$$
c_{2}(\alpha)=\frac{1}{\pi^{2} \alpha^{2}} \int_{-\infty}^{\infty} d v \cdot \log \left[\int_{0}^{\infty} \frac{1}{\Gamma(1+\alpha)}\left\{\frac{|v-z|^{\alpha}}{|v-1-\alpha|^{\alpha}}\right\} z^{\alpha} \exp (-z) d z\right]
$$

The case for $\alpha=2$, when the coefficients are normally distributed with mean zero and variance unity, had been treated by one of us in [1]. It had been found that outside the interval $-1 \leqslant x \leqslant 1$, the number of maxima and minima of these curves, on an average, does not differ substantially from the number indicated by their axis-crossings, showing thereby that the oscillations which do not cross the mean level $y=0$ are to some extent localised to the range $-1<x \leqslant 1$. This phenomenon, observed for the case $\alpha=2$, will be seen to hold good for all $\alpha$ in $0<\alpha<2$ as well. As there, it will further be shown here that most of the maxima and minima occur near $x=1$ and -1 , a fact which is true for the axis-crossings also.

We briefly discuss the coefficients $c_{1}(\alpha)$ and $c_{2}(\alpha)$ below. To begin with, $c_{1}(\alpha)$ may be expressed as

$$
c_{1}(\alpha)=\frac{1}{\pi^{2} \alpha^{2}} \int_{0}^{\infty} d v \log \left[\left\{\Gamma(1+\alpha)+(-)^{1+\alpha} \gamma(1+\alpha, v)\right\} \frac{\Gamma(1+\alpha,-v)}{\left|v^{2}-1\right|^{\alpha}}\right]
$$

where

$$
\gamma(1+\alpha, x)=\int_{0}^{x} e^{-t} t^{\alpha} d t
$$

and

$$
\Gamma(1+\alpha, x)=\Gamma(1+\alpha)-\gamma(1+\alpha, x)
$$

are the familiar incomplete Gamma functions. These shall further be expressed in terms of the confluent hypergeometric functions

$$
{ }_{1} F_{1}(a, c ; x)=\Phi(a, c ; x)=\sum_{0}^{\infty} \frac{(a)_{n}}{(c)_{n}} \frac{x^{n}}{n !}
$$

with $(a)_{0}=1$ and $(a)_{n}=a(a+1) \ldots(a+n-1)$, in the form

$$
(-)^{1+\alpha} \gamma(1+\alpha,-v)=(1+\alpha)^{-1} v^{1+\alpha} \Phi(1+\alpha, 2+\alpha ; v) \text {, }
$$

and

$$
\Gamma(1+\alpha, v)=\Gamma(1+\alpha)-(1+\alpha)^{-1} v^{1+\alpha} \Phi(1+\alpha, 2+\alpha ;-v)
$$


On integration by parts, the relation $0<\alpha \leqslant 2$ yields the asymptotic estimates (as $v \rightarrow \infty)$

$$
\begin{gathered}
\Gamma(1+\alpha, v)=e^{-v}\left\{v^{\alpha}+\alpha v^{\alpha-1}+\alpha(\alpha-1) v^{\alpha-2}\right. \\
\left.+\alpha(\alpha-1)(\alpha-2) v^{\alpha-3}+A_{1}(v) v^{\alpha-4}\right\}, \\
\gamma(1+\alpha,-v) \cdot(-1)^{1+\alpha}=e^{v}\left\{v^{\alpha}-\alpha v^{\alpha-1}+\alpha(\alpha-1) v^{\alpha-2}\right. \\
\left.-\alpha(\alpha-1)(\alpha-2) v^{\alpha-3}+A_{2}(v) v^{\alpha-4}\right\}
\end{gathered}
$$

with $\left|A_{i}(v)\right|<16$. This shows that the expression for $c_{1}(\alpha)$ is

$$
c_{1}(\alpha)=\frac{1}{\pi^{2} \alpha^{2}} \int_{0}^{\infty} \log g(v) d v
$$

where

$$
g(v)=1+\left(\alpha^{2}-\alpha\right) / v^{2}+O\left(\alpha / v^{4}\right) \quad(v \rightarrow \infty)
$$

for $\alpha \neq 1$. When $\alpha=1$ (the Cauchy case) since $\Phi(2,3 ; v)=2(1-\exp (v)+$ $v \exp (v)) / v^{2}$, we find

$$
\begin{aligned}
c_{1}(1) & =\frac{1}{\pi^{2}} \int_{0}^{\infty} \log \left[\left\{1+\frac{1}{2} v^{2} \Phi(2,3 ; v)\right\}\left\{1-\frac{1}{2} v^{2} \Phi(2,3 ;-v)\right\} /\left|v^{2}-1\right|\right] \\
& =\frac{1}{\pi^{2}} \int_{0}^{\infty} \log \frac{v-1+2 \exp (-v)}{\left|v^{2}-1\right|} d v \\
& =\frac{2}{\pi^{2}} \int_{0}^{\infty} \frac{v}{2+(v-1) \exp (v)} d v=0.185 \ldots
\end{aligned}
$$

on integration by parts and machine computation.

Now, $c_{2}(\alpha)$ may be expressed as

$$
c_{2}(\alpha)=\frac{1}{\pi^{2} \alpha^{2}} \int_{0}^{\infty} d v \log \left[\frac{v^{1+2 \alpha}\left\{I_{1 / 2+\alpha}\left(\frac{1}{2} v\right)+\pi^{-1} K_{1 / 2+\alpha}\left(\frac{1}{2} v\right)\right\} K_{1 / 2+\alpha}\left(\frac{1}{2} v\right)}{\left|v^{2}-(1+\alpha)^{2}\right|^{\alpha}}\right]
$$

where $I_{\nu}(x)$ and $K_{\nu}(x)$ are the usual Bessel functions of imaginary argument. When we use the asymptotic estimates

$$
\begin{gathered}
K_{1 / 2+\alpha}\left(\frac{1}{2} v\right) \sim\left(\frac{\pi}{v}\right)^{1 / 2} e^{-v / 2}\left[1+\frac{\alpha(1+\alpha)}{v}+\frac{\alpha\left(\alpha^{2}-1\right)(2+\alpha)}{2 v^{2}}+\ldots\right], \\
I_{1 / 2+\alpha}\left(\frac{1}{2} v\right) \sim\left(\frac{1}{\pi v}\right)^{1 / 2} e^{v / 2}\left[1-\frac{\alpha(1+\alpha)}{v}+\frac{\alpha\left(\alpha^{2}-1\right)(2+\alpha)}{2 v^{2}}+\ldots\right]
\end{gathered}
$$

we find

$$
c_{2}(\alpha)=\frac{1}{\pi^{2} \alpha^{2}} \int_{0}^{\infty} \log h(v) d v
$$


where

$$
h(v)=1+\frac{\alpha\left(1-\alpha^{2}\right)}{v^{2}}+O\left(\frac{\alpha}{v^{4}}\right) \text { as } v \rightarrow \infty \text { for } \alpha \neq 1 .
$$

This shows that the integrands $\log g(v)$ and $\log h(v)$ are uniformly expansible in power series in $\alpha$ for small positive $\alpha$ and we shall resort to these techniques below. If $\alpha=1$, i.e. if the coefficients $X_{k}$ have the Cauchy distribution, the relations

$$
\begin{gathered}
K_{3 / 2}\left(\frac{v}{2}\right)=\left(\frac{\pi e^{-v}}{v}\right)^{1 / 2}\left(1+\frac{2}{v}\right) \text { and } \\
I_{3 / 2}\left(\frac{v}{2}\right)=\left(\frac{e^{v}}{\pi v}\right)^{1 / 2}\left[1-\frac{2}{v}+e^{-v}\left(1+\frac{2}{v}\right)\right]
\end{gathered}
$$

ensure

$$
\begin{aligned}
c_{2}(1) & =\frac{1}{\pi^{2}} \int_{0}^{\infty} d v \log \left[\frac{v-2+2 e^{-v}(v+2)}{|v-2|}\right] \\
& =\frac{2}{\pi^{2}} \int_{0}^{\infty} \frac{v^{2}}{2(v+2)+(v-2) e^{v}} d v=0.269 \ldots
\end{aligned}
$$

on integration by parts and machine computation.

Next we discuss the behaviour of $c_{1}(\alpha)$ and $c_{2}(\alpha)$ as $\alpha \rightarrow 0$. We shall use the estimates

$$
\begin{aligned}
& \Gamma\left(\frac{1}{2}+\alpha\right)=\pi^{1 / 2}\left[1-(\gamma+2 \log 2) \alpha+\left\{\frac{(\gamma+2 \log 2)^{2}}{2}+\frac{\pi^{2}}{4}\right\} \alpha^{2}+\ldots\right] \\
& \Gamma(1+2 \alpha) / \Gamma(1+\alpha)=1-\gamma \alpha+\left(\frac{1}{2} \gamma^{2}+\frac{1}{4} \pi^{2}\right) \alpha^{2}+\ldots, \\
& \Gamma(-1-2 \alpha) / \Gamma(-\alpha)=-\frac{1}{2}+\left(1-\frac{1}{2} \gamma\right) \alpha-\left(2-\gamma+\frac{1}{4} \gamma^{2}+\frac{1}{8} \pi^{2}\right) \alpha^{2}+\ldots \\
& \Phi(1+\alpha, 2+\alpha ; v)=\frac{1}{v}\left[e^{v}-1+\left(\sum_{1}^{\infty} \frac{k-1}{k} \frac{v^{k}}{k !}\right) \alpha\right. \\
& \left.\quad-\left(\sum_{1}^{\infty} \frac{k-1}{k^{2}} \frac{v^{k}}{k !}\right) \alpha^{2}+\ldots\right] \\
& \Phi(1+\alpha, 2+2 \alpha ; v)=\frac{1}{v}(1+\alpha)\left[e^{v} \Phi(\alpha, 2+2 \alpha ;-v)-\Phi(\alpha, 2+2 \alpha ; v)\right], \\
& \Phi(-\alpha,-2 \alpha ; v)=\frac{1}{v}\left[e^{v} \Phi(-\alpha, 1-2 \alpha ;-v)+\Phi(-\alpha, 1-2 \alpha ; v)\right]
\end{aligned}
$$

(Kummer's relations), 


$$
\begin{aligned}
\Phi(\alpha, 2+2 \alpha ; v) & =1+\left(\sum_{1}^{\infty} \frac{1}{k} \frac{v^{k}}{(k+1) !}\right) \alpha \\
+ & \left\{\sum_{1}^{\infty}\left(1-\frac{1}{2}-\ldots-\frac{1}{k-1}-\frac{2}{k}-\frac{2}{k+1}\right) \frac{1}{k} \frac{v^{k}}{(k+1) !}\right\} \alpha^{2} \\
+\ldots, & \\
\Phi(-\alpha, 1-2 \alpha ; v) & =1-\left(\sum_{1}^{\infty} \frac{1}{k} \frac{v^{k}}{k !}\right) \alpha \\
& -\left\{\sum_{1}^{\infty}\left(1+\frac{1}{2}+\cdots+\frac{1}{k \cdot-1}+\frac{2}{k}\right) \frac{1}{k} \frac{v^{k}}{k !}\right\} \alpha^{2}+\ldots
\end{aligned}
$$

Let

$$
\begin{aligned}
\lambda \equiv \lambda(v) & =\int_{0}^{v} \frac{e^{t}-1}{t} d t \\
& =v+\frac{1}{2} \frac{v^{2}}{2 !}+\frac{1}{3} \frac{v^{3}}{3 !}+\frac{1}{4} \frac{v^{4}}{4 !}+\cdots+\frac{1}{k} \frac{v^{k}}{k !}+\ldots, \\
\mu \equiv \mu(v) & =\int_{0}^{v} \frac{\lambda(t)}{t} d t \\
& =v+\frac{1}{2^{2}} \frac{v^{2}}{2 !}+\frac{1}{3^{2}} \frac{v^{3}}{3 !}+\cdots+\frac{1}{k^{2}} \frac{v^{k}}{k !}+\ldots, \\
\nu \equiv \nu(v) & =-\int_{0}^{v} \frac{e^{t} \lambda(-t)}{t} d t=\sum_{1}^{\infty}\left(1+\frac{1}{2}+\cdots+\frac{1}{k}\right) \frac{1}{k} \frac{v^{k}}{k !},
\end{aligned}
$$

on use of the identity

$$
\sum_{1}^{\infty}\left(1+\frac{1}{2}+\cdots+\frac{1}{k}\right) \frac{x^{k}}{k !}=-e^{x} \lambda(-x) .
$$

Thus

$$
\begin{aligned}
& v \Phi(1+\alpha, 2+\alpha ; v)= e^{v}-1+\left\{e^{v}-1-\lambda(v)\right\} \alpha \\
&-\{\lambda(v)-\mu(v)\} \alpha^{2}+\ldots, \\
& v \Phi(1+\alpha, 2+2 \alpha ; v)= e^{v}-1+\left\{e^{v} \lambda(-v)-\lambda(v)+2 e^{v}-2\right\} \alpha \\
&+\left\{2 e^{v} \lambda(-v)-2 \lambda(v)+\mu(v)-e^{v} \mu(-v)+\nu(v)-e^{v} \nu(-v)\right\} \alpha^{2}+\ldots . \\
& 2 \Phi(-\alpha,-2 \alpha ; v)=e^{v}+1-\left\{e^{v} \lambda(-v)+\lambda(v)\right\} \alpha \\
&-\left\{\mu(v)+e^{v} \mu(-v)+\nu(v)+e^{v} \nu(-v)\right\} \alpha^{2}+\ldots
\end{aligned}
$$

These make 


$$
\begin{aligned}
& c_{1}(\alpha)=\frac{1}{\pi^{2} \alpha^{2}} \int_{0}^{\infty} \log \left\{P_{1}(v)+Q_{1}(v) \alpha+R_{1}(v) \alpha^{2}+\ldots\right\} d v, \\
& c_{2}(\alpha)=\frac{1}{\pi^{2} \alpha^{2}} \int_{0}^{\infty} \log \left\{P_{2}(v)+Q_{2}(v) \alpha+R_{2}(v) \alpha^{2}+\ldots\right\} d v,
\end{aligned}
$$

where $P_{1}(v)=1=P_{2}(v)$,

$$
\begin{aligned}
Q_{1}(v)= & Q_{2}(v)=-\lambda(-v) e^{v}-\lambda(v) e^{-v} \\
& +\log v\left(2-e^{v}-e^{-v}\right)-\gamma\left(e^{v}+e^{-v}\right)-\log \left|1-v^{2}\right| .
\end{aligned}
$$

Now $\int_{0}^{\infty} Q_{1}(v) d v=\int_{0}^{\infty} Q_{2}(v) d v=0$ on integration by parts and the use of the estimates (as $v \rightarrow \infty)$

$$
\begin{gathered}
\lambda(v) \sim e^{v}\left(\frac{1}{v}+\frac{1}{v^{2}}+\frac{2}{v^{3}}+\ldots\right), \mu(v) \sim e^{v}\left(\frac{1}{v^{2}}+\frac{2}{v^{3}}+\ldots\right), \\
\lambda(-v) \sim \log v-\gamma-e^{-v}\left(\frac{1}{v}-\frac{1}{v^{2}}-\ldots\right), \\
\mu(-v) \sim-\frac{1}{2}(\log v)^{2}-\gamma \log v-\ldots, \quad 5>-v(-v)>1 .
\end{gathered}
$$

(For $v>0$,

$$
\left.v \lambda(v)>e^{v}-1-v+\frac{v^{2}}{2}, \quad v^{2} \mu(v)>e^{v}-1-\frac{v^{2}}{2} .\right)
$$

Thus

$$
c_{1}(\alpha)=\frac{1}{\pi^{2}} \int_{0}^{\infty}\left[R_{1}(v)-\frac{1}{2} Q_{1}^{2}(v)\right] d v+O(\alpha)
$$

as $\alpha \rightarrow 0^{+}$, where now writing $\varepsilon, \iota, \lambda, \bar{\varepsilon}, \bar{\lambda}, \mu, \bar{\mu}$ in place of $e^{v}, \log v, \lambda(v), e^{-v}$, $\mu(v), \mu(-v)$ and $\lambda(-v)$ for short, we have

$$
\begin{aligned}
R_{1}-Q_{1}^{2}= & \varepsilon\left(\bar{\mu}-\bar{\lambda}_{\iota}+\bar{\varepsilon} \frac{\iota^{2}}{2}+\frac{\iota^{2}}{2}+\frac{\gamma^{2}}{2}+\frac{\pi^{2}}{12}\right)-\frac{1}{2} \varepsilon \varepsilon(\bar{\varepsilon} \iota-\iota-\bar{\lambda}-\gamma)^{2} \\
& +\bar{\varepsilon}\left(\mu-\lambda_{\iota}+\varepsilon \frac{\iota^{2}}{2}+\frac{\iota^{2}}{2}+\frac{\gamma^{2}}{2}+\frac{\pi^{2}}{12}\right)-\frac{1}{2} \bar{\varepsilon} \bar{\varepsilon}(\varepsilon \iota-\iota-\lambda-\gamma)^{2} .
\end{aligned}
$$

On use of the estimates

$$
\begin{aligned}
\mu(v) \cdot e^{-v} & =1 / v^{2}+2 / v^{3}+\ldots \\
-\mu(-v) & \sim \frac{1}{2}(\log v)^{2}+\gamma \log v+\ldots
\end{aligned}
$$

as $v \rightarrow \infty$ and integration by parts, we obtain $c_{1}\left(0^{+}\right)=0.25$. Similar calculations yield $c_{2}\left(0^{+}\right)=0.25$. Machine calculations indicate that as $\alpha$ increases to 2 through positive values, $c_{1}(\alpha)$ decreases from 0.25 to $0.159 \ldots$ and $c_{2}(\alpha)$ increases from 0.25 to $0.275 \ldots$ Hence $c_{1}(\alpha)<c_{2}(\beta)$ for any two $\alpha$ and $\beta$, and there is an extra maximum in the interval $-1<x<1$ for 
stable laws. The same is, of course, true for the case of minima also. We may anticipate the above phenomenon from the following considerations. The maxima and minima are determined by the equation $\Sigma X_{k} k x^{k-1}=0$ with probability one, and as $k$ increases, $x^{k-1}$ decreases for any $x$ in $0<x<1$, so that the product $k \cdot x^{k-1}$ has a complex structure. But for $1<x<\infty$, $k x^{k-1}$ increases with $k$; i.e. shows the same tendency as in the last interval. The $x^{k}$ being the elements of the equation $\Sigma x_{k} x^{k}=y$ determining the algebraic curve, the oscillations result solely from the oscillations of the coefficients alone. This suggests why the maxima and the minima should be nearly as many as the crossings of the mean level in the ranges $1<|x|<\infty$. But in the intervals $0<|x|<1$, the oscillations result both from the coefficient $X_{k}$ and the elements $k x^{k-1}$, so that there are an extra number of oscillations in this case.

2. We begin with certain observations: If $\left(x_{0}, y_{0}\right)$ is a point of maxima for the curve $y=X_{0}+X_{1} x+\cdots+X_{n} x^{n}$, then $\left(x_{0},-y_{0}\right)$ is a point of minima for the curve $y=-X_{0}-X_{1} x-X_{2} x^{2}-\cdots-X_{n} x^{n}$ and vice versa, and since the distribution function of the $X_{k}$ 's is symmetric, both the curves belong to the family. As such, the family of curves $y=P(x)$ will have, on an average, the same number of maxima as they have minima. Also the curve $y=X_{0}-X_{1} x+X_{2} x^{2}-\cdots+(-)^{n} X_{n} x^{n}$ in the range $-\infty<x<0$ is the reflection about the $y$-axis of the curve $y=X_{0}+X_{1} x+X_{2} x^{2}$ $+\cdots+X_{n} x^{n}$ in the range $0<x<\infty$. Further $\operatorname{Pr} \cdot\left(P^{\prime}(x)=0\right.$ at $\left.x=0\right)$ $=0$. Thus we need only consider the range $x: 0<x<\infty$.

Now a curve $y=P(x)$ may have stationary points of inflection. However, the totality of such curves forms a negligible portion of the whole family. To see this, we may denote the $(n-1)$ roots of $P^{\prime}(x)=0$ as $\rho_{1}, \rho_{2}, \rho_{3}, \ldots, \rho_{n-1}$. Then

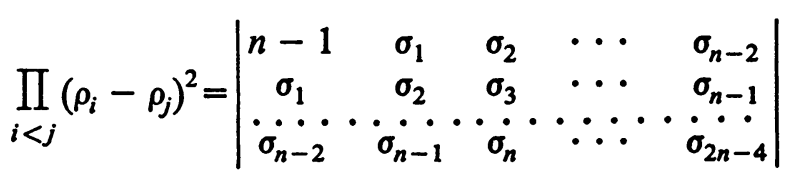

where

$$
\sigma_{i}=\sum_{1}^{n-1}\left(\rho_{k}\right)^{i}=\rho_{1}^{i}+\cdots+\rho_{n-1}^{i} .
$$

Further the $\sigma_{i}$ can be developed as polynomials in the coefficients of $P^{\prime}(x)$ through Newton's identities in the theory of symmetric polynomials. Thus $\Pi\left(\rho_{i}-\rho_{j}\right)^{2}$ is a polynomial of the $X_{k}$ 's, i.e. $\Pi_{i<j}\left(\rho_{i}-\rho_{j}\right)^{2}=$ $D\left(X_{0}, X_{1}, \ldots, X_{n}\right)$. Now $x=r$ (real) is a stationary point of inflection for the curve $y=P(x)$ if and only if $P^{\prime}(r)=P^{\prime \prime}(r)=0$, i.e. $r$ is a multiple zero 
of $P^{\prime}(x)$, and, $r$ is equal with $\rho_{i}$ and $\rho_{j}$ for two different $i$ and $j$. This causes $D\left(X_{0}, X_{1}, \ldots, X_{n}\right)$ to vanish, an event which can have probability zero only, since $D$ involves only constant coefficients and the $X_{k}$ 's, and the last set possesses identical continuous distribution functions (cf. [3, p. 183]). As such, we find that with probability one, the total number of maxima and minima of $y=P(x)$ is given by the number of real zeros of $P^{\prime}(x)$.

3. Let $M_{n}(a, b ; \alpha)$ denote the number of maxima of the curve $y=P(x)$ in $a<x<b$, and let $N_{n}(a, b ; \alpha)$ denote the number of real zeros of $x P^{\prime}(x)$. The foregoing arguments establish the following relation for the expectations of $M_{n}$ and $N_{n}$, viz:

$$
2 E M_{n}(a, b ; \alpha)=E N_{n}(a, b ; \alpha) .
$$

We put $Q(x)=x \cdot P^{\prime}(x)=\Sigma_{1}^{n} k X_{k} x^{k}$. Our methods will closely follow those of [2] and [8]. We put $\psi_{\varepsilon}(x)=1$ if $-\varepsilon<x<\varepsilon$ and $\psi_{\varepsilon}(x)=0$ if $|x|>\varepsilon$. Now from Lemma 1 of Kac [5], we have

$$
N_{n}(a, b ; \alpha)=\lim _{\varepsilon \rightarrow 0} \frac{1}{2 \varepsilon} \int_{a}^{b} \psi_{\varepsilon}(Q(x)) \cdot\left|Q^{\prime}(x)\right| d x .
$$

Hence

$$
E N_{n}(a, b ; \alpha)=\lim _{\varepsilon \rightarrow 0} \frac{1}{2 \varepsilon} \int_{a}^{b} E\left\{\psi_{\varepsilon}(Q(x)) \cdot|Q(x)|\right\} d x .
$$

The combined variable $\left(Q(x), Q^{\prime}(x)\right)$ has characteristic function

$$
f(z, w)=E\left[\exp \left\{i Q(x) z+i Q^{\prime}(x) w\right\}\right] .
$$

Thus the probability density $p(\xi, \eta)$ for $Q(x)=\xi$ and $Q^{\prime}(x)=\eta$ is given by the Fourier inversion formula

$$
p(\xi, \eta)=\frac{1}{(2 \pi)^{2}} \int_{-\infty}^{\infty} \int_{-\infty}^{\infty} \exp (-i \xi z-i \eta \omega) f(z, w) d z d w
$$

and the chance that $\xi<Q(x)<\xi+d \xi$ and $\eta<Q^{\prime}(x)<\eta+d \eta$ hold simultaneously is $p(\xi, \eta) d \xi d \eta$. As the $x$ 's vary, both $Q$ and $Q^{\prime}$ assume values from $-\infty$ to $\infty$, so that

$$
E\left\{\psi_{\varepsilon}(Q) \cdot\left|Q^{\prime}\right|\right\}=\int_{-\infty}^{\infty} \int_{-\infty}^{\infty} \psi_{\varepsilon}(\xi) \cdot|\eta| \cdot p(\xi, \eta) d \xi d \eta
$$

Let us write

$$
F(\xi)=\int_{-\infty}^{\infty}|\eta| p(\xi, \eta) d \eta .
$$

Now $F(\xi)$ is continuous in $\xi$ and therefore

$$
\lim _{\varepsilon \rightarrow 0} \frac{1}{2 \varepsilon} E\left\{\psi_{\varepsilon}(Q) \cdot\left|Q^{\prime}\right|\right\}=\lim _{\varepsilon \rightarrow 0} \frac{1}{2 \varepsilon} \int_{-\varepsilon}^{\varepsilon} F(\xi) d \xi=F(0) .
$$


Thus, since all the functions in the integrand below are bounded, we have

$$
E N_{n}(a, b ; \alpha)=\lim _{\varepsilon \rightarrow 0} \frac{1}{2 \varepsilon} \int_{a}^{b} E\left\{\psi_{\varepsilon}(Q(x)) \cdot\left|Q^{\prime}(x)\right|\right\} d x=\int_{a}^{b} F(0) d x
$$

and we obtain the Kac-Rice formula

$$
E N_{n}(a, b ; \alpha)=\int_{a}^{b} d x \int_{-\infty}^{\infty}|\eta| \cdot p(0, \eta) d \eta
$$

Putting

$$
Q(x)=\sum_{1}^{n} X_{k} a_{k} \text { and } Q^{\prime}(x)=\sum_{1}^{n} X_{k} b_{k}
$$

we find that

$$
f(z, \omega)=\exp \left(-\sum_{1}^{n}\left|a_{k} z+b_{k} \omega\right|^{\alpha}\right)
$$

so that

$$
p(0, y)=\frac{1}{(2 \pi)^{2}} \int_{-\infty}^{\infty} d \omega \int_{-\infty}^{\infty} \exp (-i y \omega) \cdot \exp \left(-\sum_{1}^{n}\left|a_{k} z+b_{k} \omega\right|^{\alpha}\right) d z
$$

Hence for $\varepsilon>0$, we have

$$
\begin{aligned}
& \int_{-\infty}^{\infty}|\eta| \exp (-\varepsilon|\eta|) \cdot p(0, \eta) d \eta \\
& =\Re\left[\frac{1}{\pi^{2}} \int_{0}^{\infty} \frac{d \omega}{(\varepsilon+i \omega)^{2}} \int_{-\infty}^{\infty} \exp \left\{-\sum_{1}^{n}\left|a_{k} z+b_{k} \omega\right|^{\alpha}\right\} d z .\right.
\end{aligned}
$$

Let $A$ and $B$ be arbitrary nonzero constants. When we take $a_{k}$ and $b_{k}$ to be any constants in $k$, independent of $x$, then the probability density $p(\xi, \eta)$ corresponding to $\xi=\Sigma X_{k} a_{k}=A \bar{a}$ and $\eta=\Sigma X_{k} b_{k}=B \bar{b}$ is zero. Further, given $A$ and $B$, the constants $a_{k}$ and $b_{k}$ can be so chosen that $\bar{a}$ and $\bar{b}$ have a stable distribution function with characteristic exponent $\alpha$. But then the left-hand side of the above relation is zero. Thus

$$
0=\Re \frac{1}{\pi^{2}} \int_{0}^{\infty} \frac{d \omega}{(\varepsilon+i \omega)^{2}} \int_{-\infty}^{\infty} \exp \left\{-|A z+B \omega|^{\alpha}\right\} d z
$$

Subtracting (9) from (8) we obtain the convergent integral (10) below in the limit as $\varepsilon \rightarrow 0$ provided we make the special choice

$$
A^{\alpha}=\sum_{1}^{n} a_{k}^{\alpha}, \quad B A^{\alpha-1}=\sum b_{k} a_{k}^{\alpha-1}
$$

with $a_{k}>0$ for each $k$. We get 


$$
\begin{aligned}
\int_{-\infty}^{\infty}|\eta| p(0, \eta) d \eta=\frac{1}{\pi^{2}} \int_{0}^{\infty} \frac{d \omega}{\omega^{2}} \int_{-\infty}^{\infty} & \exp \left\{-|A z+B \omega|^{\alpha}\right\} \\
& -\exp \left\{-\sum\left|a_{k} z+b_{k} \omega\right|^{\alpha}\right\} d z .
\end{aligned}
$$

We put $z=u \omega$ and use Frullani's theorem to integrate on $\omega$. For the special choice of $A$ and $B$ given above, we obtain the right-hand side of (10), after certain reductions, in the form of the convergent integral (cf. [8, (13)])

$$
f_{n}(x ; \alpha)=\frac{1}{\pi^{2} \alpha} \int_{-\infty}^{\infty} \log \left[\sum_{1}^{n}\left|a_{k} u+b_{k}\right|^{\alpha} /|A u+B|^{\alpha}\right] d u .
$$

Let $a>0$ and $a<x<b$. We put $a_{k}=k x^{k}$ and $b_{k}=k^{2} x^{k-1}$ to obtain

$$
f_{n}(x ; \alpha)=\frac{1}{\pi^{2} \alpha x} \int_{-\infty}^{\infty} \log g_{n}(u, x ; \alpha) d u
$$

where

$$
g_{n}(u, x ; \alpha)=\sum_{k=1}^{n}|u-k|^{\alpha} \theta_{k}\left(x^{\alpha}\right) /\left|u-\bar{\theta}\left(x^{\alpha}\right)\right|^{\alpha}
$$

with

$$
\theta_{k}(x)=k^{\alpha} x^{k} /\left(\sum_{j=1}^{n} j^{\alpha} x^{j}\right) \text { and } \bar{\theta}(x)=\sum_{1}^{n} k \theta_{k}(x),
$$

We have

$$
E M_{n}(a, b ; \alpha)=\frac{1}{2} E N_{n}(a, b ; \alpha) .
$$

Further, since the coefficients $X_{k}$ are symmetrically distributed about zero, the coefficients of $x^{k}$ in $Q(x)$ and $Q(-x)$ have the same distribution, so that $E N_{n}(-r, 0 ; \alpha)=E N_{n}(0, r ; \alpha)$ and

$$
E M_{n}(-\infty, \infty ; \alpha)=\lim _{\alpha \rightarrow 0^{+}, b \rightarrow \infty} E N_{n}(a, b ; \alpha),
$$

and it therefore suffices to evaluate $E N_{n}(a, b ; \alpha)$ for $b>a>0$. By the foregoing we have, since $a_{k} \geqslant k a^{k}>0$,

$$
\begin{aligned}
\operatorname{EN}_{n}(a, b ; \alpha) & =\int_{a}^{b} d x \int_{-\infty}^{\infty}|\eta| \cdot p(0, \eta) d \eta=\int_{a}^{b} f_{n}(x ; \alpha) d x \\
& =\frac{1}{\pi^{2} \alpha} \int_{a}^{b} \frac{d x}{x} \int_{-\infty}^{\infty} \log g_{n}(u, x ; \alpha) d u
\end{aligned}
$$

with $g_{n}$ as above. We make the substitution $\tau=x^{\alpha}$ to obtain

$$
E N_{n}(a, b ; \alpha)=\frac{1}{\pi^{2} \alpha} \int_{a^{\alpha}}^{b^{\alpha}} \frac{d \tau}{\tau} \int_{-\infty}^{\infty} \frac{1}{\alpha} \log h(u, \tau ; \alpha) d u,
$$

where 


$$
h(u, \tau ; \alpha)=\sum_{k=1}^{n}|u-k|^{\alpha} \theta_{k}(\tau) /|u-\bar{\theta}(\tau)|^{\alpha} .
$$

We shall make use of Hölder's inequality [4, p. 26, 2.9.1] for mean values with weights $\theta_{k}(\tau)$. Since $\Sigma \theta_{k}(\tau)=1,0<\alpha<2$, we thus have

$$
\left[\sum_{k=1}^{n}|u-k|^{\alpha} \theta_{k}(\tau)\right]^{1 / \alpha}<\left[\sum_{k=1}^{n}(u-k)^{2} \theta_{k}(\tau)\right]^{1 / 2} \text {. }
$$

At this point it is necessary to use our results in [2]. Let $c_{1}, c_{2}, c_{3}, \ldots$ be a sequence of mutually independent, normally distributed random variables with mathematical expectation zero and variance unity. Let $\rho>0$ and let $N_{n}^{*}(a, b)$ denote the number of real zeros of

$$
P^{*}(x)=c_{1} x+2^{\rho} c_{2} x^{2}+3^{\rho} c_{3} x^{3}+\cdots+n^{\rho} c_{n} x^{n} .
$$

In that work [2], we have shown that

$$
E N_{n}^{*}(a, b)=\int_{a}^{b} \frac{d x}{2 \pi^{2} x} \int_{-\infty}^{\infty} \log g_{n}^{*}(u, x) d u
$$

where

$$
g_{n}^{*}(u, x)=\left\{\sum_{1}^{n}(u-k)^{2} \phi_{k}\left(x^{2}\right)\right\} /\left\{u-\phi\left(x^{2}\right)\right\}^{2}
$$

with

$$
\phi_{k}(\tau)=k^{2 \rho \tau^{k}} /\left(\sum_{j=1}^{n} j^{2 \rho \tau^{j}}\right) \text { and } \phi(\tau)=\sum_{1}^{n} k \phi_{k}(\tau) .
$$

Putting $x^{2}=\tau$, this gives

$$
E N_{n}^{*}\left(a^{\alpha / 2}, b^{\alpha / 2}\right)=\frac{1}{2 \pi^{2}} \int_{a^{\alpha}}^{b^{\alpha}} \frac{d \tau}{\tau} \int_{-\infty}^{\infty} \log h^{*}(u, \tau) d u
$$

where

$$
h^{*}(u, \tau)=\left\{\sum_{k=1}^{n}(u-k)^{2} \phi_{k}(\tau)\right\} /\{u-\phi(\tau)\}^{2} .
$$

From now on we make the substitution $2 \rho=\alpha$ so that $\phi_{k}(\tau)$ and $\phi(\tau)$ become, respectively, equal with $\theta_{k}(\tau)$ and $\bar{\theta}(\tau)$. With this substitution (18) becomes

$$
\left[\sum|u-k|^{\alpha} \theta_{k}(\tau)\right]^{1 / \alpha}<\left[\sum(u-k)^{2} \phi_{k}(\tau)\right]^{1 / 2}
$$

that is,

$$
[h(u, \tau ; \alpha)]^{1 / \alpha}<\left[h^{*}(u, \tau)\right]^{1 / 2}
$$


Hence we obtain

$$
\begin{aligned}
E N_{n}(a, b ; \alpha) & =\frac{1}{\pi^{2} \alpha} \int_{a^{\alpha}}^{b^{\alpha}} \frac{d \tau}{\tau} \int_{-\infty}^{\infty} \frac{1}{\alpha} \log h(u, \tau ; \alpha) d u \\
& <\frac{1}{\pi^{2} \alpha} \int_{a^{\alpha}}^{b^{\alpha}} \frac{d \tau}{\tau} \int_{-\infty}^{\infty} \frac{1}{2} \log h^{*}(u, \tau) d u \\
& =(2 / \alpha) E N^{*}\left(a^{\alpha / 2}, b^{\alpha / 2}\right) .
\end{aligned}
$$

We put $\delta=\exp \left(-(\log n)^{1 / 3}\right)$ and $T=(\log n)^{1 / 2}$. Using (2.5)-(2.7) of [2], we obtain

$$
\begin{aligned}
E N_{n}(1+\delta, \infty ; \alpha) & <\frac{2}{\alpha} E N^{*}\left((1+\delta)^{\alpha / 2}, \infty\right)<\frac{2}{\alpha} E N^{*}(1+3 \delta, \infty) \\
& =O(\log \delta)=O\left\{(\log n)^{1 / 3}\right\} \\
E N_{n}(0,1-\delta ; \alpha) & <\frac{2}{\alpha} E N^{*}\left(0,(1-\delta)^{\alpha / 2}\right)<\frac{2}{\alpha} E N^{*}\left(0,1-\frac{\alpha \delta}{4}\right) \\
& =O(\log \delta)=O\left\{(\log n)^{1 / 3}\right\}
\end{aligned}
$$

and

$$
\begin{aligned}
E N_{n}\left(1-\frac{T}{n},\right. & \left.1+\frac{T}{n} ; \alpha\right)<\frac{2}{\alpha} E N^{*}\left(\left(1-\frac{T}{n}\right)^{\alpha / 2},\left(1+\frac{T}{n}\right)^{\alpha / 2}\right) \\
& <\frac{2}{\alpha} E N^{*}\left(1-\frac{T}{n}, 1+\frac{T}{n}\right)=O(T)=O\left((\log n)^{1 / 2}\right) .
\end{aligned}
$$

Our next aim is to estimate $E N_{n}(1+T / n, 1+\delta)$ and $E N_{n}(1-\delta, 1-$ $T / n)$. We proceed to evaluate the first one and set $\tau=e^{t / n}, u=n v / t$ in (16) and (17). Thus

$$
\begin{aligned}
E N_{n}\left(1+\frac{T}{n}, 1+\delta ; \alpha\right) & =\int_{1+T / n}^{1+\delta} f_{n}(x ; \alpha) d x \\
& =\frac{1}{\pi^{2} \alpha} \int_{T_{1}}^{n \delta_{1}} \frac{d t}{t} \int_{-\infty}^{\infty} \frac{1}{\alpha} \log l(v, t ; \alpha) d v
\end{aligned}
$$

where

$$
T_{1}=n \log (1+T / n) \sim T \text { and } \delta_{1}=\log (1+\delta) \sim \delta
$$

with

$$
l(v, t ; \alpha)=\frac{\Sigma_{k}|n v / t-k|^{\alpha} k^{\alpha} \exp (k t / n) /\left(\Sigma_{j} j^{\alpha} \exp (j t / n)\right)}{\left|n v / t-\left(\Sigma_{k} k^{1+\alpha} \exp (k t / n)\right) /\left(\Sigma_{j} j^{\alpha} \exp (j t / n)\right)\right|^{\alpha}} .
$$

We shall convert $l(v, t ; \alpha)$ into a form suitable for evaluation by asymptotic methods. With this end in view, we multiply the numerator and the 
denominator of (25) by $(t / n)^{\alpha}$ and obtain

$$
\begin{aligned}
l(v, t ; \alpha) & =\frac{\left[\Sigma_{k}|v-k t / n|^{\alpha}(k t / n)^{\alpha} \exp (k t / n)\right] /\left[\Sigma_{j}(j t / n)^{\alpha} \exp (j t / n)\right]}{\left|v-\left(\Sigma_{k}(k t / n)^{1+\alpha} \exp (k t / n)\right) /\left(\Sigma_{j}(j t / n)^{\alpha} \exp (j t / n)\right)\right|^{\alpha}} \\
& =\frac{\left[\Sigma_{k}|v-k t / n|^{\alpha} \cdot|v-\bar{\theta}|^{-\alpha} \exp (k t / n) \cdot(t / n)\right] \cdot(k t / n)^{\alpha}}{\left[\Sigma_{j}(j t / n)^{\alpha} \exp (j t / n) \cdot(t / n)\right]}
\end{aligned}
$$

where

$$
\bar{\theta}=\frac{\left[\Sigma_{k}(k t / n)^{1+\alpha} \exp (k t / n) \cdot(t / n)\right]}{\left[\Sigma_{j}(j t / n)^{\alpha} \exp (j t / n) \cdot(t / n)\right]} .
$$

Now for $\beta>0$,

$$
\int_{0}^{t-t / n} y^{\beta} \exp (y) d y<\sum_{j}\left(\frac{j t}{n}\right)^{\beta} \exp \left(\frac{j t}{n}\right) \cdot\left(\frac{t}{n}\right)<\int_{0}^{t / n} y^{\beta} \exp (y) d y,
$$

so that for $T_{1}<t<n \delta$, we fine

$$
\bar{\theta} \sim\left(\int_{1}^{t} y^{1+\alpha} \exp (y) d y\right) /\left(\int_{1}^{t} y^{\alpha} \exp (y) d y\right),
$$

that is, $\bar{\theta}=t-1+\alpha^{2} / t-\ldots$ and $t-\bar{\theta}=1-\gamma(t)$ where $|\gamma(t)|<$ (constant) $/ t$ for all $t$ in $\left(T_{1}, n \delta_{1}\right)$. Now as above in (19), Hölder's inequality yields $\alpha^{-1} \log l(v, t ; \alpha)<\frac{1}{2} \log l(v, t ; 2)$. Also

$$
\begin{aligned}
l(v, t ; 2) & =\frac{\left[\Sigma_{k}(v-k t / n)^{2} \exp (k t / n)(k t / n)_{\Delta}^{2}\right]}{(v-\bar{\theta})^{2}\left[\exp (k t / n)(k t / n)^{2}\right]} \\
& =1+5 /(v-\bar{\theta})^{2}+\ldots
\end{aligned}
$$

by actual reduction and division. Further

$$
\begin{aligned}
l(v, t ; \alpha) & =\frac{\left[\Sigma_{k}|v-k t / n|^{\alpha}(k t / n)^{\alpha} \exp (k t / n) \cdot t / n\right]}{\left[\Sigma_{k}|v-\bar{\theta}|^{\alpha}(k t / n)^{\alpha} \exp (k t / n) \cdot t / n\right]} \\
& =\frac{\left[\Sigma_{k}|v-t+(n-k) t / n|^{\alpha}(k t / n)^{\alpha} \exp ((k-n) t / n) \cdot t / n\right]}{\left[\Sigma_{k}|v-\bar{\theta}|^{\alpha}(k t / n)^{\alpha} \exp ((k-n) t / n) t / n\right]} \\
& =\frac{\left[\Sigma_{m}|m t / n-V|^{\alpha}(t-m t / n)^{\alpha} \exp (-m t / n)(t / n)\right]}{\left[\Sigma_{m}|1-\gamma(t)-V|^{\alpha}(t-m t / n)^{\alpha} \exp (-m t / n) t / n\right]} \\
& =\lambda(V, t ; \alpha)(\mathrm{say}) ;
\end{aligned}
$$


on putting $t-v=V$; and we obtain

$$
E N_{n}\left(1+\frac{T}{n}, 1+\delta ; \alpha\right)=\frac{1}{\pi^{2} \alpha} \int_{T_{1}}^{n \delta_{1}} \frac{d t}{t} \int_{-\infty}^{\infty} \frac{1}{\alpha} \log \lambda(V, t ; \alpha) d V .
$$

Also, by (29)

$$
l(v, t ; 2)=\lambda(V, t ; 2)=1+5\left(1-\gamma_{0}(t) / t-V\right)^{-2}+\ldots
$$

where $\left|\gamma_{0}(t)\right|<A$, an absolute constant for all $t$ in $\left(T_{1}, n \delta_{1}\right)$. Thus for any $L$,

$$
\begin{aligned}
\frac{1}{\alpha} \int_{|V|>L} & \log \lambda(V, t ; \alpha) d V<\frac{1}{2} \int_{|V|>L} \log \lambda(V, t ; 2) d V \\
& <\frac{1}{2} \int_{|V|>L} \log \left\{1+\frac{10}{\left(V-1+\gamma_{0}(t) / t\right)^{2}}\right\} d V=O\left(\frac{1}{L}\right) .
\end{aligned}
$$

For $|V|<L$, we have

$$
\lambda(V, t ; \alpha)=\frac{\Sigma_{m} \mid m t / n-\eta^{\alpha}\left(\sum_{\nu=0}^{\infty}\left(\begin{array}{l}
\alpha \\
\nu
\end{array}\right)(-m / n)^{\nu}\right) \exp (-m t / n) \cdot(t / n)}{\Sigma_{m}|V-1-\gamma(t)|^{\alpha}\left(\sum_{\nu=0}^{\infty}\left(\begin{array}{l}
\alpha \\
\nu
\end{array}\right)(-m / n)^{\nu}\right) \exp (-m t / n) \cdot(t / n)},
$$

where $\left(\begin{array}{l}\alpha \\ \nu\end{array}\right)$ are the binomial coefficients if $\alpha$ is nonintegral, $\left(\begin{array}{l}\alpha \\ \nu\end{array}\right)=0$ if $\alpha$ is an integer and $\nu>\alpha$. Hence

$$
\begin{aligned}
\lambda(V, t ; \alpha) & =\left(\sum_{\nu=0}^{\infty} a_{\nu} t^{-\nu}\right) /\left(\sum_{\nu=0}^{\infty} b_{\nu} t^{-\nu}\right) \\
& =\frac{a_{0}}{b_{0}}+\frac{b_{0} a_{1}-a_{0} b_{1}}{b_{0}^{2}}\left(\frac{1}{t}\right)+\ldots
\end{aligned}
$$

with

$$
a_{\nu}=\sum_{m}\left|\frac{m}{n} t-V\right|^{\alpha}\left(\begin{array}{l}
\alpha \\
\nu
\end{array}\right)\left(-\frac{m}{n}\right)^{\nu} \exp \left(-\frac{m t}{n}\right) \cdot\left(\frac{t}{n}\right)
$$

and

$$
b_{\nu}=\sum_{m}|V-1+\gamma(t)|^{\alpha}\left(\begin{array}{l}
\alpha \\
\nu
\end{array}\right)\left(-\frac{m}{n}\right)^{\nu} \exp \left(-\frac{m t}{n}\right) \cdot\left(\frac{t}{n}\right)
$$

These considerations make 


$$
\begin{aligned}
& E N_{n}\left(1+\frac{T}{n}, 1+\delta ; \alpha\right)=\frac{1}{\pi^{2} \alpha} \int_{T_{1}}^{n \delta_{1}} \frac{d t}{t} \int_{-\infty}^{\infty} \frac{1}{\alpha} \log \lambda(V, t ; \alpha) d V \\
& =\frac{1}{\pi^{2} \alpha} \int_{T_{1}}^{n \delta_{1}} \frac{d t}{t}\left\{\int_{-L}^{L} \frac{1}{\alpha} \log \left(\frac{a_{0}}{b_{0}}+\frac{b_{0} a_{1}-a_{0} b_{1}}{b_{0}^{2}} \cdot \frac{1}{t}+\cdots\right) d V+O\left(\frac{1}{L}\right)\right\} \\
& =\frac{1}{\pi^{2} \alpha} \int_{T_{1}}^{n \delta_{1}} \frac{d t}{t}\left\{\int_{-L}^{L} \frac{1}{\alpha}\left(\log \frac{a_{0}}{b_{0}}+\frac{A_{1}}{t}+\frac{B_{1}}{t^{2}}+\cdots\right) d V+O\left(\frac{1}{L}\right)\right\} \\
& =\frac{1}{\pi^{2} \alpha} \int_{T_{1}}^{n \delta_{1}} \frac{d t}{t}\left[\left\{\int_{-L}^{L} \frac{1}{\alpha} \log \frac{\Sigma_{m}|V-m t / n|^{\alpha} \exp (-m t / n) \cdot t / n}{\Sigma_{m}|V-\bar{\theta}|^{\alpha} \exp (-m t / n) \cdot t / n}\right\}\right.
\end{aligned}
$$

The last integral in the braces is the same as that which occurs in the work of Logan and Shepp [8] with $V$ and $t$ in place of their $v$ and $x$. When we evaluate this by their methods we find that

$$
E N_{n}(1+T / n, 1+\delta ; \alpha)=\left[c_{1}(\alpha)+\varepsilon_{n}\right] \log n
$$

where

$$
c_{1}(\alpha)=\frac{1}{\pi^{2} \alpha^{2}} \int_{-\infty}^{\infty} d V \log \int_{0}^{\infty}\left[\frac{|V-y|^{\alpha}}{|V-1|^{\alpha}}\right] \exp (-y) d y
$$

and $\varepsilon_{n} \rightarrow 0$ as $n \rightarrow \infty$. (A similar approximation has been examined closely by us below using an alternative method.)

This proves that outside the interval $(-1,1)$, the number of maxima and minima of the curves $y=P(x)$, on an average, does not differ substantially from the number indicated by their axis-crossings, a result which we had established in [1] for the case $\alpha=2$.

4. Lastly we are to estimate $E N_{n}(1-\delta, 1-T / n ; \alpha)$. By the substitution $u=n v / t$ and $\tau=\exp (-t / n)$ in (16) and (17), this is seen to be equal to

$$
\frac{1}{\pi^{2} \alpha} \int_{T_{0}}^{n \delta_{0}} \frac{d t}{t} \int_{-\infty}^{\infty} \frac{1}{\alpha} \log p(v, t ; \alpha) d v
$$

where

$$
T_{0}=-n \log (1-T / n), \quad \delta_{0}=-\log (1-\delta),
$$

and 


$$
p(v, t ; \alpha)=\frac{\Sigma_{k}|v-k t / n|^{\alpha}(k t / n)^{\alpha} \exp (-k t / n) \cdot(t / n)}{\Sigma_{k}|v-\bar{\theta}|^{\alpha}(k t / n)^{\alpha} \exp (-k t / n) \cdot(t / n)}
$$

with

$$
\begin{aligned}
\bar{\theta} & =\frac{\Sigma_{j}(j t / n)^{1+\alpha} \exp (-j t / n) \cdot(t / n)}{\Sigma_{j}(j t / n)^{\alpha} \exp (-j t / n) \cdot(t / n)} \\
& =(1+\alpha)+O(t \cdot \exp (-t / 2)) .
\end{aligned}
$$

(A proof of the last can be found on pp. 60-61 of [2].) As before we can prove that

$$
\int_{-\infty}^{\infty} \frac{1}{\alpha} \log p(v, t ; \alpha) d v=\int_{-L}^{L} \frac{1}{\alpha} \log p(v, t ; \alpha) d v+O(1 / L) .
$$

For $|v|<L$ and $T_{0}<t<n \delta_{0}$, we find, on the use of the celebrated EulerMaclaurin sum formula (cf. e.g., [6, pp. 520-524]) for the numerator and the denominator below, and estimate (41) for $\bar{\theta}$, that

$$
\begin{aligned}
& p(v, t ; \alpha)=\frac{\Sigma_{k}|v-k t / n|^{\alpha}(k t / n)^{\alpha} \exp (-k t / n) \cdot(t / n)}{\Sigma_{k}|v-\bar{\theta}|^{\alpha}(k t / n)^{\alpha} \exp (-k t / n) \cdot(t / n)} \\
& =\frac{\int_{0}^{t}|v-z|^{\alpha} z^{\alpha} \exp (-z) d z+O\left(t^{2 \alpha} \exp (-t)\right)}{\int_{0}^{t}|v-1-\alpha|^{\alpha} z^{\alpha} \exp (-z) d z+O\left(t^{2 \alpha} \exp (-t)\right)+O\left(t^{2 \alpha} \exp (-\alpha t / 2)\right)} \\
& =\frac{\int_{0}^{\infty}|v-z|^{\alpha} z^{\alpha} \exp (-z) d z+O\left(t^{2 \alpha} \exp (-t)\right)}{\int_{0}^{\infty}|v-1-\alpha|^{\alpha} z^{\alpha} \exp (-z) d z+O\left(t^{2 \alpha} \exp (-\alpha t / 2)\right)} \\
& =\frac{\int_{0}^{\infty}|v-z|^{\alpha} z^{\alpha} \exp (-z) d z}{\int_{0}^{\infty}|v-1-\alpha|^{\alpha} z^{\alpha} \exp (-z) d z}+O\left(t^{2 \alpha} \exp (-\alpha t / 2)\right)
\end{aligned}
$$

as $t \rightarrow \infty$. Now, as in (19)

$$
\alpha^{-1} \log p(v, t ; \alpha)<\frac{1}{2} \log p(v, t ; 2),
$$

and $T_{0}$ can be taken so large that $p(v, t ; 2)>1$ for all $t>T_{0}$ so that 


$$
\begin{aligned}
& \frac{1}{\pi^{2} \alpha} \int_{T_{0}}^{n \delta_{0}} \frac{d t}{t} \int_{-L}^{L} \frac{1}{\alpha} \log p(v, t ; \alpha) d v \\
& \quad \leqslant \frac{1}{\pi^{2} \alpha} \int_{T_{0}}^{n \delta_{0}} \frac{d t}{t} \int_{-L}^{L} \frac{1}{2} \log p(v, t ; 2) d v \\
& \quad<\frac{1}{2 \alpha \pi^{2}} \int_{T_{0}}^{n \delta_{0}} \frac{d t}{t} \int_{-\infty}^{\infty} \log p(v, t ; 2) d v
\end{aligned}
$$

and the last integral has been explicitly evaluated by us in [2, pp. 62-63]. Hence we can make $L$ approach infinity below to obtain from (39) and (42),

$$
\begin{aligned}
& E N_{n}\left(1-\delta, 1-\frac{T}{n} ; \alpha\right)=\frac{1}{\pi^{2} \alpha} \int_{T_{0}}^{n \delta_{0}} \frac{d t}{t} \int_{-\infty}^{\infty} \frac{1}{\alpha} \log p(v, t ; \alpha) d v \\
& =\frac{1}{\pi^{2} \alpha} \int_{T_{0}}^{n \delta_{0}} \frac{d t}{t}\left[\int_{-L}^{L} \frac{1}{\alpha} \log p(v, t ; \alpha) d v+O\left(\frac{1}{L}\right)\right] \\
& =\frac{1}{\pi^{2} \alpha^{2}} \int_{T_{0}}^{n \delta_{0}} \frac{d t}{t} \int_{-\infty}^{\infty} d v \\
& \quad \cdot \log \left[\int_{0}^{\infty} \frac{1}{\Gamma(1+\alpha)} \frac{|v-z|^{\alpha}}{|v-1-\alpha|^{\alpha}} z^{\alpha} \exp (-z) d z+\varepsilon_{n}\right] \\
& =c_{2} \log n+\eta,
\end{aligned}
$$

where $|\eta|<\eta_{n} \log n$ with $\varepsilon_{n}, \eta_{n}$ approaching zero as $n$ approaches infinity and

$$
c_{2}=c_{2}(\alpha)=\int_{-\infty}^{\infty} d v \log \left[\int_{0}^{\infty} \frac{1}{\Gamma(1+\alpha)}\left\{\frac{|v-z|^{\alpha}}{|v-1-\alpha|^{\alpha}}\right\} z^{\alpha} \exp (-z) d z\right]
$$

This, combined with (21), (22), (23) and (38) shows that

$$
E M_{n} \sim\left(c_{1}+c_{2}\right) \log n=c \log n \quad \text { as } n \rightarrow \infty,
$$

completing our assertion.

\section{REFERENCES}

1. M. Das, The average number of maxima of a random algebraic curve, Proc. Cambridge Philos. Soc. 65 (1969), 741-753.

2. __ Real zeros of a class of random algebraic polynomial, J. Indian Math. Soc. 36 (1972), $53-63$.

3. B. V. Gnedenko and A. N. Kolmogorov, Limit distributions for sums of independent random variables, Addison-Wesley, Reading, Mass., 1954.

4. G. H. Hardy, J. E. Littlewood and G. Polya, Inequalities, Cambridge Univ. Press, 1952.

5. M. Kac, On the average number of real roots of a random algebraic equation, Bull. Amer. Math. Soc. 49 (1943), 314-320. 
6. K. Knopp, Theory and application of infinite series, Blackie, London, 1957.

7. B. F. Logan and L. A. Shepp, Real zeros of random polynomials, Proc. London Math. Soc. (3) 18 (1968), 29-35.

8. Real zeros of random polynomials. II, Proc. London Math. Soc. (3) 18 (1968), $308-314$.

Departmgent of Mathematics, Fakir Mohan College, Balasore-756001, Orissa, India 